\title{
Why Autonomous Driving Is So Hard: The Social Dimension of Traffic
}

\author{
Hannah Pelikan
}

\section{Conference paper}

Cite this conference paper as:

Pelikan, H. Why Autonomous Driving Is So Hard: The Social Dimension of Traffic, In (eds), HRI '21 Companion, March 8-11, 2021, Boulder, CO, USA, : IEEE Computer Society; 2021, pp. 81-85. ISBN: 9781450382908

DOI: https://doi.org/10.1145/3434074.3447133

ACM/IEEE International Conference on Human-Robot Interaction, 2167-2148, No.

Copyright: IEEE Computer Society

\section{http://www.ieee.org/}

The self-archived postprint version of this journal article is available at Linköping University Institutional Repository (DiVA):

http://urn.kb.se/resolve?urn=urn:nbn:se:liu:diva-173058 


\title{
Why Autonomous Driving Is So Hard: The Social Dimension of Traffic
}

\author{
Hannah R. M. Pelikan \\ hannah.pelikan@liu.se \\ Linköping University \\ Linköping, Sweden
}

\begin{abstract}
Smooth traffic presupposes fine coordination between different actors, such as pedestrians, cyclists and car drivers. When autonomous vehicles join regular traffic, they need to coordinate with humans on the road. Prior work has often studied and designed for interaction with autonomous vehicles in structured environments such as traffic intersections. This paper describes aspects of coordination also in less structured situations during mundane maneuvers such as overtaking. Taking an ethnomethodological and conversation analytic approach, the paper analyzes video recordings of self-driving shuttle buses in Sweden. Initial findings suggest that the shuttle buses currently do not comply with cyclists' expectations of social coordination in traffic. The paper highlights that communication and coordination with human road users is crucial for smooth flow of traffic and successful deployment of autonomous vehicles also in less structured traffic environments.
\end{abstract}

\section{CCS CONCEPTS}

- Human-centered computing $\rightarrow$ Field studies; Collaborative and social computing.

\section{KEYWORDS}

autonomous vehicle, shuttle bus, cyclist, conversation analysis, ethnomethodology, field study, mobile interaction

\section{ACM Reference Format:}

Hannah R. M. Pelikan. 2021. Why Autonomous Driving Is So Hard: The Social Dimension of Traffic. In Companion of the 2021 ACM/IEEE International Conference on Human-Robot Interaction (HRI '21 Companion), March 8-11, 2021, Boulder, CO, USA. ACM, New York, NY, USA, 5 pages. https: //doi.org/10.1145/3434074.3447133

\section{INTRODUCTION}

When we humans encounter another vehicle or cyclist on the road, we typically coordinate our movements smoothly to pass one another. We communicate our intentions through eye gaze, bodily orientation, pace, and gestures (e.g. holding out the right arm to indicate that we are going to turn right on a bike). In most motor

Permission to make digital or hard copies of all or part of this work for personal or classroom use is granted without fee provided that copies are not made or distributed for profit or commercial advantage and that copies bear this notice and the full citation on the first page. Copyrights for components of this work owned by others than the author(s) must be honored. Abstracting with credit is permitted. To copy otherwise, or republish, to post on servers or to redistribute to lists, requires prior specific permission and/or a fee. Request permissions from permissions@acm.org.

HRI '21 Companion, March 8-11, 2021, Boulder, CO, USA

(c) 2021 Copyright held by the owner/author(s). Publication rights licensed to ACM ACM ISBN 978-1-4503-8290-8/21/03...\$15.00

https://doi.org/10.1145/3434074.3447133 vehicles, we use lights (indicators) and sounds (honks) to communicate with each other $[2,12]$. As autonomous vehicles encounter other road users, they too need to communicate what they are going to do next. This has mostly been explored in physically structured locations such as crosswalks and intersections, with specific scenarios like a pedestrian who needs to know whether the car will stop for them and whether it is safe to pass in front of it [1, 4, 13, 21, 24].

However, coordination on the road is not limited to particular landmarks and scenarios. Ordinary driving practices such as changing lanes, overtaking and letting oneself be overtaken also involve careful coordination $[5,12]$. This becomes especially important when autonomous vehicles need to maneuver close to cyclists and pedestrians. This work reports on initial findings from a demonstration project in Linköping, Sweden, in which self-driving shuttle buses by two different manufacturers are currently being tested on the university campus and its surroundings. The study is part of a project that focuses on everyday driving in spaces that are not so clearly structured and where coordination may simply involve walking beside or overtaking an autonomous shuttle bus without getting in each other's way. Taking an approach that is rooted in conversation analysis and ethnomethodology (EMCA, see 3.2), traffic interaction is seen as inherently social $[25,29]$. The paper makes two contributions. First, it demonstrates that coordination is not only an issue in structured environments such as traffic intersections (where safety drivers currently confirm that the bus is safe to go) but also in mundane driving practices such as overtaking. Second, the paper discusses how self-driving shuttle buses in public transport could coordinate smoothly with other road users and what this implies for robots in live traffic.

\section{RELATED WORK}

Research on autonomous vehicles has mostly focused on cars, exploring interaction design and how other road users behave around them (e.g. [9, 18, 20, 21, 24, 29]). Visuals, sounds, and vibro-tactile feedback have been explored as a means to communicate a vehicle's intentions to other road users such as pedestrians [6, 9, 13, 21] and cyclists [11]. Some work has argued that interaction should be implicit and movement may be sufficient for communicating whether an autonomous car is going to stop at an intersection $[21,23]$. Following this argumentation, additional visual and auditory cues should only be used when people get too close to an autonomous car [24]. Others stress that explicit cues are beneficial in communicating whether it is safe to cross $[6,13]$, and to switch lanes [11] when an autonomous car is approaching.

User studies with vehicles of other kinds than cars are still scarce. Along with electric bikes and scooters [27], shuttle buses are one possible solution for last-mile transport, connecting users' homes 
with larger hubs and offering a means of transport for people with impaired mobility. Being shared and often part of public transport, buses encounter different driving situations than cars and regularly maneuver close to pedestrians and cyclists. Prior research on interaction with autonomous buses has largely focused on passengers, i.e. immediate users [15-17, 22, 30]. An ethnographic field study has demonstrated how safety drivers negotiate with car drivers where to overtake the shuttle bus [7] and pedestrians' and cyclists' perspectives have been taken into account when designing means of interaction for autonomous shuttle buses [1, 14, 28]. However, we still know little about how unprotected traffic participants like cyclists and pedestrians actually behave around shuttle buses.

EMCA studies on human interaction have demonstrated that traffic is highly coordinated: Human drivers slow down to offer gaps for others to pass through [10] and negotiate overtaking through reciprocal adjustments of speed (slowing down/speeding up) and trajectory (e.g. moving to one side of a lane) [5]. During such maneuvers, human drivers make use of gestures, but also honks [12] and headlights [10] to communicate with each other.

\section{METHOD}

The studied shuttle buses are built by different manufacturers, EasyMile $^{1}$ and Navya ${ }^{2}$, and are demonstrated in the Ride the Future project $^{3}$, which explores future traffic solutions. Both shuttle buses are electric and navigate based on data from LIDAR and GPS sensors. When approaching and leaving a bus stop and when breaking down strongly, the shuttle buses play a "ding" sound (the sounds are different on each shuttle). Each shuttle bus fits up to 15 passengers.

The shuttle buses serve a round of 8 stops during daytime and rides are for free. The buses drive with a speed of $10-15 \mathrm{~km} / \mathrm{h}(6-$ $9 \mathrm{mph}$ ), and a full round takes 20 minutes. While the buses technically could ride faster, they are driving slow for safety reasons. On a full route, the shuttle buses drive on (1) a public road, (2) a smaller road next to a parking lot, as well as (3) the main campus avenue, which is reserved for pedestrians and cyclists (service vehicles are occasionally present). The campus avenue is loosely separated into a pedestrian and a bike lane, and the buses drive in the area that is designated as a bike lane. Colored dots with a shuttle bus symbol have been added in between the bike symbols painted on the road, indicating that both groups should use this area. The shuttle buses drive on a fixed trajectory, from which they only divert after stopping and calculating a small deviation to get around obstacles.

The buses move autonomously, and are advertised as being able to drive without a driver on board (level 4 autonomy). However, in the demonstration project there is always a safety driver on board. The safety driver is tasked with monitoring the driving and taking manual control over the vehicle whenever a dangerous situation occurs. The safety driver also gives their okay before the bus passes through larger intersections. Safety drivers in this project are employees of the local public transport provider. They have several years of experience in driving buses and/or trams, and received special training to operate the autonomous shuttle buses.

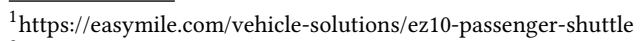

${ }^{2}$ https://navya.tech/en/solutions/moving-people/

${ }^{3}$ https://ridethefuture.se
}

\subsection{Data Collection and Ethics}

First field observations were carried out in January 2020, as the project was newly started. The research team gradually started to video record interaction around the shuttle buses, at first from stationary positions on the road and subsequently with up to four GoPros placed inside the buses. During the rides, the author discussed with the safety drivers how they experience the buses' capacities to communicate, based on their comparable expertise in driving buses and trams. Due to reduced campus activities during the COVID-19 pandemic, there was generally less traffic on campus when most of the recordings were made. While we observed more incidents prior to the pandemic and during times of partial return to campus, we continued to observe interesting interactions with the bus even when the campus was less frequented.

All participants have given consent to be recorded inside the bus. While recording in public is within Sweden's law, we wanted to ensure that recordings were obtained in an ethical manner. To indicate whenever the bus was recording video, camera signs were placed on the bus. Passengers were informed about the video recording just before entering the bus, and cameras facing inwards were switched off when passengers did not consent to being recorded.

\subsection{Analytic Methodology}

The ethnomethodologic and conversation analytic perspective [8, $19,26]$ is grounded in an inductive, micro-sociological approach. Working on video recordings of real-world interaction, EMCA scrutinizes social interaction and its organization in fine detail. In this project, focus lies on everyday mundane interaction with the shuttle buses and moments in which their planned movements are disturbed. Fieldnotes and video data are reviewed for such disturbances and relevant clips are saved for later analysis in collections. EMCA regards actions as holistic and situated multimodal Gestalts [19]. A honk for instance can only be understood by looking at the current situation, what has happened in the environment just (milli)seconds before, and what is happening right after the honk [12]. An important tool for such moment-by-moment analysis are detailed transcripts of interaction, in which audible phenomena, movement, gaze, gesture and engagements with material objects are noted down in detail. Rather than assessing what is happening from a technical perspective (did the sensor detect the cyclist in time?) or inferring intents (did the cyclist intend to stop?), EMCA analysis relies on road users' visible behavior and their understanding of actions, which they display in the situated context (e.g. holding out a hand, starting to turn, flashing a light, braking). EMCA regards actions as situated and dynamically adjusted rather than indifferently following a pre-determined plan [26], therefore it is important to scrutinize what is happening at each moment. The transcripts, which may resemble extremely detailed (theater) scripts enable the researcher to pinpoint the emergent sequentiality - how one action follows from what happened before and how it projects possible next actions. Transcripts also serve as a resource for joint analysis and are validated in data sessions, in which videos are jointly viewed and alternative interpretations discussed. Data from this project have been presented at three separate data sessions with 12,4 , and 11 EMCA researchers, respectively. EMCA approaches typically present a few exemplary cases to illustrate general patterns. 

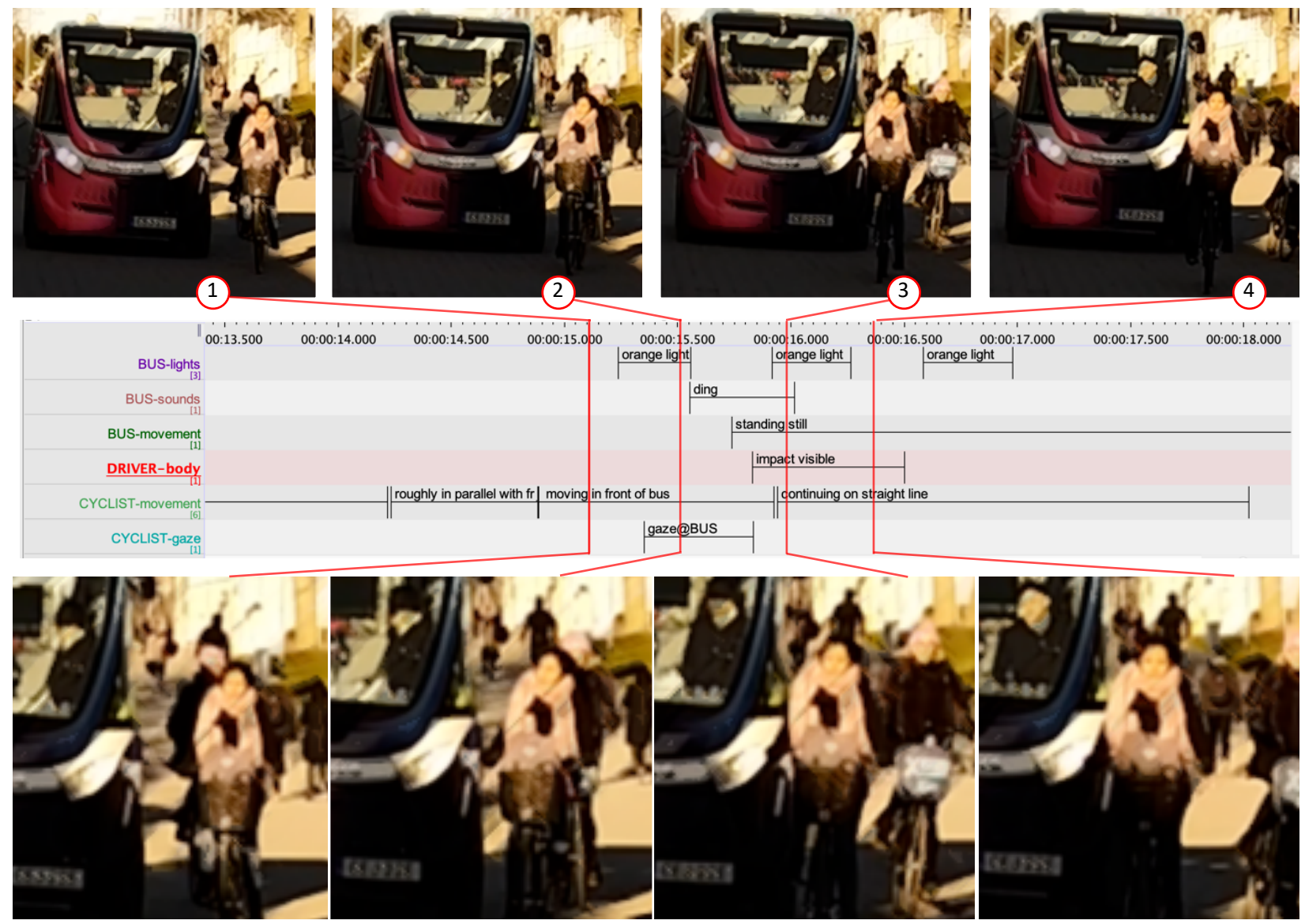

Figure 1: A cyclist causes emergency braking. Middle: timeline in seconds with annotated observations. Top: Frame grabs (time codes indicated by red lines in the timeline). Bottom: Close ups of the top pictures.

\section{INITIAL FINDINGS}

Both on the regular road and in the mixed campus environment, the buses face difficulties when coordinating with other road users. By default, the buses elicit emergency braking when they cannot handle a situation. This happens particularly often when the buses are overtaken by cars and cyclists who are driving at a faster speed. Figure 1 illustrates a typical pattern of a cyclist overtaking the bus. As the cyclist is finishing the overtaking maneuver, the bus responds with a sudden brake. Starting to pull in just in front of the bus, the overtaking cyclist gets rather close to the bus front (see pictures 1 and 2). This causes the bus to flash an orange light (pic. $2-3$ ), to play a "ding" sound and to sharply brake to a full halt. The cyclist gazes towards the bus (pic. 2) before moving in front of it (pic. 3) but does not turn around for another look when the bus is emergency braking. While the cyclist continues their trajectory, the bus remains halted in the middle of the road for several seconds.

In the close ups, the impact on the safety driver's body is visible. $\mathrm{He}$ is initially standing $90^{\circ}$ tilted towards the driving direction (pic. 1-2) but is flung towards the front window by the sudden braking (pic. 4). As can be imagined from the pictures, and as also highlighted in the researcher's ethnographic fieldnotes, the sudden brakes are not very comfortable for the people inside the bus and can potentially lead to injuries.

In addition to the overtaking cyclist, the bus also interacts with other people who are simultaneously present on the road. They too are affected by the abrupt braking. Another cyclist (visible just behind the first cyclist, pic. 1-4) immediately steers away from the bus when it starts braking (visible in pic. 3). Moving away from the bus, the cyclist crosses into the oncoming traffic and ultimately has to jump off the bike to prevent a crash with an oncoming cyclist. Even if this is not directly involving the bus, it demonstrates that sudden emergency braking can be consequential for unprotected traffic participants like cyclists.

Even nine months after the buses started operating, similar situations continue to occur. A text added to the back of the bus saying "I can brake strongly - thank you for keeping the distance :)" in Swedish does not seem to prevent them. Figure 2 illustrates a situation in which a cyclist is overtaking the bus on the public road. An oncoming car slows down as the cyclist approaches, and accelerates after the cyclist has passed. The cyclist continues smoothly after overtaking the bus. In contrast, the shuttle bus ends up braking in the same way as in Figure 1. It remains halted for several seconds, thereby becoming a potential obstacle for other vehicles. 




Figure 2: A cyclist is overtaking the shuttle bus. While the oncoming car is slowing down to let the cyclist pass, the bus first continues with the same speed. As the cyclist starts moving in front of the bus, the bus triggers sharp braking.

In the data, human road users coordinate their actions in careful ways, by slowing down, speeding up, or adjusting their trajectories, thereby maintaining a smooth flow. As concerns the buses, they are not participating in coordination at the same level of detail and mostly continue on their fixed trajectory with the same speed. While they may slow down slightly in some cases, their braking often comes too abrupt. As documented in Figure 1, the shuttle bus does not just stop but also flashes its lights and plays a sound when braking. However, being elicited just as the bus is initiating braking, these signals come too late to work as warnings. They do not warn the cyclists not to get closer and are too sudden to give passengers and safety drivers time to tense up their bodies for the impact. Rather, they merely provide a visual and auditory counterpart to the actual braking. It seems that the sounds could be heard as honks at the person who has already passed, but cyclists rarely turn around to see why the shuttle is playing a sound. It is therefore questionable whether the sounds and lights as used in the shuttle buses help cyclists to learn to keep a bigger distance.

\section{IMPLICATIONS}

First insights from this project highlight that coordination occurs as soon as several vehicles and people share the road. EMCA research has demonstrated that interaction in traffic is inherently social and people constantly coordinate their actions [25, 29]. Humans communicate through horns, indicators and gestures but also by slowing down or moving faster $[2,5,10,12]$. The autonomous shuttle buses are not exempt from this and people seem to expect them to participate in this coordination. Pulling in closely in front of the shuttle buses as exemplified in Figures 1 and 2, cyclists display expectations that the bus would slow down to let them overtake, or divert from its fixed trajectory. While abrupt emergency braking prevents crashes, it does not contribute to a smooth traffic flow and may even be harmful for passengers and surrounding road users.

If we want robots to participate smoothly in regular traffic, they need to be designed for movement and continuous coordination with humans even in mundane situations. Traffic involves highly complex social communication and there is no time out from negotiating with others, as we constantly coordinate, slowing down and speeding up [3, 5, 10] and using gestures [7]. One way to address this would be to monitor and predict the trajectories of cyclists and other road users in more detail. Shuttle buses could start slowing down when cyclists are detected pulling out behind the bus, or speed up to indicate implicitly that they should not be overtaken. As humans do not follow pre-determined plans but adjust their actions dynamically to the evolving situation [26], such predictions will have to be constantly updated.

When autonomous shuttle buses cannot divert from their route and are unable to react by slowing down early, it may be necessary to communicate this inability clearly to surrounding road users. Driving in marked lanes could be one solution [14], but a requirement for separate lanes might narrow the range of spaces in which shuttle buses may deployed. A safety driver in the study explained how he reacts in regular public transport: To warn that a tram cannot divert from its route, he starts braking and honking long before a potential crash. We plan to further research how shuttle buses could communicate that their capabilities for coordination are limited. It needs to be further explored to what extent designs for autonomous cars can be applied, since accountability and expectations may be different for shared public vehicles.

A central issue in automation of public transport involves the possibility for one human operator to manage entire fleets of vehicles remotely, taking over control if an autonomous vehicle is confronted with a situation which it cannot handle. As local and situated coordination is constantly necessary, handling coordination tasks remotely will likely be challenging. As this paper demonstrates, traffic conditions that require joint coordination are not limited to specific spatial structures such as roundabouts and intersections and may be hard to predict as they can appear anytime and anywhere on the road, even during mundane driving situations.

\section{CONCLUSION}

This paper reports initial findings of how cyclists interact with autonomous shuttle buses during everyday rides on a Swedish university campus. Video recordings collected throughout one year demonstrate that the buses stop repeatedly in between their regular stops, as other road users get too close. The data shows that the shuttle buses often fail to coordinate their movements with other people who are normally present in regular traffic. While it is well known that autonomous vehicles need to interact with other road users to achieve a smooth flow of traffic, many studies are limited to structured areas such as crossings and intersections. The initial findings presented here stress the importance of studying interaction with autonomous vehicles in real world traffic, throughout entire rides, particularly also aspects of driving that appear utterly mundane. To be part of a smooth traffic flow, autonomous vehicles need to constantly coordinate with those present on the road and collaborate in joint maneuvers.

\section{ACKNOWLEDGMENTS}

I would like to express my gratitude to my advisors Prof. Leelo Keevallik and Prof. Mathias Broth for their guidance on this project. I thank the MOBSIN, SIS, and AI in Interaction data session participants for their feedback. Special thanks to the safety drivers and the Ride the Future team for welcoming me on the buses. This work is funded by the Swedish Research Council, project no. 2016-00827. 


\section{REFERENCES}

[1] Marc-Philipp Böckle, Anna Pernestål Brenden, Maria Klingegård, Azra Habibovic, and Martijn Bout. 2017. SAV2P: Exploring the Impact of an Interface for Shared Automated Vehicles on Pedestrians' Experience. In Proceedings of the 9th International Conference on Automotive User Interfaces and Interactive Vehicular Applications Adjunct (Oldenburg, Germany) (AutomotiveUI '17). Association for Computing Machinery, New York, NY, USA, 136-140. https: //doi.org/10.1145/3131726.3131765

[2] Mathias Broth, Jakob Cromdal, and Lena Levin. 2018. Showing where you're going. Instructing the accountable use of the indicator in live traffic. International fournal of Applied Linguistics 28, 2 (2018), 248-264. https://doi.org/10.1111/ijal. 12194

[3] Barry Brown and Eric Laurier. 2017. The Trouble with Autopilots: Assisted and Autonomous Driving on the Social Road. Association for Computing Machinery, New York, NY, USA, 416-429. https://doi.org/10.1145/3025453.3025462

[4] Chia-Ming Chang, Koki Toda, Daisuke Sakamoto, and Takeo Igarashi. 2017. Eyes on a Car: An Interface Design for Communication between an Autonomous Car and a Pedestrian. In Proceedings of the 9th International Conference on Automotive User Interfaces and Interactive Vehicular Applications (Oldenburg, Germany) (AutomotiveUI '17). Association for Computing Machinery, New York, NY, USA, 65-73. https://doi.org/10.1145/3122986.3122989

[5] Arnulf Deppermann, Eric Laurier, Lorenza Mondada, Mathias Broth, Jakob Cromdal, Elwys De Stefani, Pentti Haddington, Lena Levin, Maurice Nevile, and Mirka Rauniomaa. 2018. Overtaking as an interactional achievement: video analyses of participants' practices in traffic. Gesprächsforschung 19 (2018), 1131. http://www.gespraechsforschung-online.de/fileadmin/dateien/heft2018/gadeppermann.pdf

[6] Debargha Dey, Kai Holländer, Melanie Berger, Berry Eggen, Marieke Martens, Bastian Pfleging, and Jacques Terken. 2020. Distance-Dependent EHMIs for the Interaction Between Automated Vehicles and Pedestrians. Association for Computing Machinery, New York, NY, USA, 192-204. https://doi.org/10.1145/3409120.3410642

[7] Grace Eden, Benjamin Nanchen, Randolf Ramseyer, and Florian Evéquoz. 2017 On the Road with an Autonomous Passenger Shuttle: Integration in Public Spaces. In Proceedings of the 2017 CHI Conference Extended Abstracts on Human Factors in Computing Systems (Denver, Colorado, USA) (CHI EA '17). Association for Computing Machinery, New York, NY, USA, 1569-1576. https://doi.org/10.1145/ 3027063.3053126

[8] Charles Goodwin. 2017. Co-Operative Action. Cambridge University Press, Cambridge, UK. https://doi.org/10.1017/9781139016735

[9] Azra Habibovic, Victor Malmsten Lundgren, Jonas Andersson, Maria Klingegård, Tobias Lagström, Anna Sirkka, Johan Fagerlönn, Claes Edgren, Rikard Fredriksson, Stas Krupenia, Dennis Saluäär, and Pontus Larsson. 2018. Communicating Intent of Automated Vehicles to Pedestrians. Frontiers in Psychology 9 (2018), 1336. https://doi.org/10.3389/fpsyg.2018.01336

[10] Pentti Haddington and Mirka Rauniomaa. 2013. Interaction Between Road Users: Offering Space in Traffic. Space and Culture 17, 2 (dec 2013), 176-190. https://doi.org/10.1177/1206331213508498

[11] Ming Hou, Karthik Mahadevan, Sowmya Somanath, Ehud Sharlin, and Lora Oehlberg. 2020. Autonomous Vehicle-Cyclist Interaction: Peril and Promise. In Proceedings of the 2020 CHI Conference on Human Factors in Computing Systems. Association for Computing Machinery, New York, NY, USA, 1-12. https://doi org/10.1145/3313831.3376884

[12] Eric Laurier, Daniel Muñoz, Rebekah Miller, and Barry Brown. 2020. A Bip, a Beeeep, and a Beep Beep: How Horns Are Sounded in Chennai Traffic. Research on Language and Social Interaction 53, 3 (2020), 341-356. https://doi.org/10.1080/ 08351813.2020.1785775

[13] Karthik Mahadevan, Sowmya Somanath, and Ehud Sharlin. 2018. Can Interfaces Facilitate Communication in Autonomous Vehicle-Pedestrian Interaction?. In Companion of the 2018 ACM/IEEE International Conference on Human-Robot Interaction (Chicago, IL, USA) (HRI '18). Association for Computing Machinery, New York, NY, USA, 309-310. https://doi.org/10.1145/3173386.3176909

[14] Natasha Merat, Tyron Louw, Ruth Madigan, Marc Wilbrink, and Anna Schieben. 2018. What externally presented information do VRUs require when interacting with fully Automated Road Transport Systems in shared space? Accident Analysis \& Prevention 118 (2018), 244 - 252. https://doi.org/10.1016/j.aap.2018.03.018

[15] Alexandra Millonig and Peter Fröhlich. 2018. Where Autonomous Buses Might and Might Not Bridge the Gaps in the 4 A's of Public Transport Passenger Needs: A Review. In Proceedings of the 10th International Conference on Automotive User Interfaces and Interactive Vehicular Applications (Toronto, ON, Canada) (AutomotiveUI '18). Association for Computing Machinery, New York, NY, USA, 291-297. https://doi.org/10.1145/3239060.3239079
[16] Alexander G. Mirnig, Magdalena Gärtner, Vivien Wallner, Sandra Trösterer, Alexander Meschtscherjakov, and Manfred Tscheligi. 2019. Where Does It Go? A Study on Visual On-Screen Designs for Exit Management in an Automated Shuttle Bus. In Proceedings of the 11th International Conference on Automotive User Interfaces and Interactive Vehicular Applications (Utrecht, Netherlands) (AutomotiveUI '19). Association for Computing Machinery, New York, NY, USA, 233-243. https://doi.org/10.1145/3342197.3344541

[17] Alexander G. Mirnig, Vivien Wallner, Magdalena Gärtner, Alexander Meschtscherjakov, and Manfred Tscheligi. 2020. Capacity Management in an Automated Shuttle Bus: Findings from a Lab Study. In 12th International Conference on Automotive User Interfaces and Interactive Vehicular Applications (Virtual Event, DC, USA) (AutomotiveUI '20). Association for Computing Machinery, New York, NY, USA, 270-279. https://doi.org/10.1145/3409120.3410665

[18] Nicole Mirnig, Nicole Perterer, Gerald Stollnberger, and Manfred Tscheligi. 2017. Three Strategies for Autonomous Car-to-Pedestrian Communication: A Survival Guide. In Proceedings of the Companion of the 2017 ACM/IEEE International Conference on Human-Robot Interaction (Vienna, Austria) (HRI '17). Association for Computing Machinery, New York, NY, USA, 209-210. https: //doi.org/10.1145/3029798.3038402

[19] Lorenza Mondada. 2019. Contemporary issues in conversation analysis: Embodiment and materiality, multimodality and multisensoriality in social interaction. Journal of Pragmatics 145 (2019), 47-62. https://doi.org/10.1016/j.pragma.2019. 01.016

[20] Dylan Moore, Rebecca Currano, Michael Shanks, and David Sirkin. 2020. Defense Against the Dark Cars: Design Principles for Griefing of Autonomous Vehicles. In Proceedings of the 2020 ACM/IEEE International Conference on Human-Robot Interaction (Cambridge, United Kingdom) (HRI '20). Association for Computing Machinery, New York, NY, USA, 201-209. https://doi.org/10.1145/3319502.3374796

[21] Dylan Moore, Rebecca Currano, G. Ella Strack, and David Sirkin. 2019. The Case for Implicit External Human-Machine Interfaces for Autonomous Vehicles. In Proceedings of the 11th International Conference on Automotive User Interfaces and Interactive Vehicular Applications (Utrecht, Netherlands) (AutomotiveUI '19). Association for Computing Machinery, New York, NY, USA, 295-307. https: //doi.org/10.1145/3342197.3345320

[22] Karl Rehrl and Cornelia Zankl. 2018. Digibus@: results from the first self-driving shuttle trial on a public road in Austria. European Transport Research Review 10, 2 (21 Nov 2018), 51. https://doi.org/10.1186/s12544-018-0326-4

[23] Malte Risto, Colleen Emmenegger, Erik Vinkhuyzen, Melissa Cefkin, and James D. Hollan. 2017. Human-Vehicle Interfaces: The Power of Vehicle Movement Gestures in Human Road User Coordination. In Proceedings of the Ninth International Driving Symposium on Human Factors in Driver Assessment, Training and Vehicle Design (Manchester Village, Vermont, June 26-29). Public Policy Center, University of Iowa, Iowa City, IA, 186-192. https://doi.org/10.17077/drivingassessment. 1633

[24] Dirk Rothenbücher, Jamy Li, David Sirkin, Brian Mok, and Wendy Ju. 2016. Ghost driver: A field study investigating the interaction between pedestrians and driverless vehicles. In 2016 25th IEEE International Symposium on Robot and Human Interactive Communication (RO-MAN). IEEE, New York, NY, USA, 795-802. https://doi.org/10.1109/ROMAN.2016.7745210

[25] Elwys De Stefani, Mathias Broth, and Arnulf Deppermann. 2019. On the road: Communicating traffic. Language and Communication 65 (2019), 1-6. https: //doi.org/10.1016/j.langcom.2018.04.009

[26] Lucy A. Suchman. 1987. Plans and Situated Actions: The Problem of HumanMachine Communication. Cambridge University Press, Cambridge, UK.

[27] Sylvaine Tuncer and Barry Brown. 2020. E-Scooters on the Ground: Lessons for Redesigning Urban Micro-Mobility. In Proceedings of the 2020 CHI Conference on Human Factors in Computing Systems (Honolulu, HI, USA) (CHI '20). Association for Computing Machinery, New York, NY, USA, 1-14. https://doi.org/10.1145/ 3313831.3376499

[28] Himanshu Verma, Guillaume Pythoud, Grace Eden, Denis Lalanne, and Florian Evéquoz. 2019. Pedestrians and Visual Signs of Intent: Towards Expressive Autonomous Passenger Shuttles. Proc. ACM Interact. Mob. Wearable Ubiquitous Technol. 3, 3, Article 107 (Sept. 2019), 31 pages. https://doi.org/10.1145/3351265

[29] Erik Vinkhuyzen and Melissa Cefkin. 2016. Developing Socially Acceptable Autonomous Vehicles. Ethnographic Praxis in Industry Conference Proceedings 2016, 1 (2016), 522-534. https://doi.org/10.1111/1559-8918.2016.01108

[30] Philipp Wintersberger, Anna-Katharina Frison, and Andreas Riener. 2018. Man vs. Machine: Comparing a Fully Automated Bus Shuttle with a Manually Driven Group Taxi in a Field Study. In Adjunct Proceedings of the 10th International Conference on Automotive User Interfaces and Interactive Vehicular Applications (Toronto, ON, Canada) (AutomotiveUI '18). Association for Computing Machinery, New York, NY, USA, 215-220. https://doi.org/10.1145/3239092.3265969 